\title{
MEG Weak Source Imaging Methods Based on Functional Connectivity
}

\author{
Ting $\mathrm{WU}^{1,{ }^{*}}$, Yuan $\mathrm{CUI}^{2}$, Zhi-guo ZHANG ${ }^{2}$, Jun-peng ZHANG ${ }^{2}$ and Tian-zi JIANG ${ }^{1}$ \\ ${ }^{1}$ Key Laboratory for Neurolnformation of the Ministry of Education, School of Life Science \\ and Technology, University of Electronic Science and Technology of China, Chengdu \\ 610054, China \\ ${ }^{2}$ Department of Medical Information Engineering, Sichuan University, Chengdu, China \\ ${ }^{*}$ Corresponding author
}

Keywords: Magnetoencephalography, Functional Connectivity, Epilepsy.

\begin{abstract}
Beam former, as a class of Magnetoencephalography (MEG) source imaging methods, is widely used. However, it has difficulty in locating weak electromagnetic sources within brain. To solve this problem, this paper attempts to develop a method for MEG weak Source Imaging. Firstly classical beam former is used to extract the source intensities and waveforms. Secondly the strongest source is taken as the seed points and calculate correlation between seed points and other voxel points to obtain Functional Connectivity (FC) images. Finally, overlying the correlation on the anatomical image to get source imaging. Such method is successfully applied to imaging bilateral primary auditory sources in the case of classical beam former fails to locate weak source which is ipsilateral to the auditory stimulus. Such method is of significance in basic and clinical research. For example, it can be used to locate eloquent cortices of epilepsy patients. Identifying these cortices is critical for them to make preoperative planning.
\end{abstract}

\section{Introduction}

Magnetoencephalography (MEG) is a functional neuroimaging technique for mapping brain activity by recording magnetic fields produced by electrical currents occurring naturally in the brain, using very sensitive magnetometers. Brain electrical activity will produce a magnetic field perpendicular to the direction of currents, the relationship between them follows right-hand rule. Permeability in biological tissue and the vacuum is almost the same, so the scalp and skull can hardly lead to the distortion of the magnetic field [1], which is an advantage of MEG over Electroencephalogram(EEG). In addition, MEG can provide high spatial resolution. MEG as a non-invasive detection technology, can detect very weak magnetic field, in the order of between fT and pT $\left(1 \mathrm{pT}=10^{-12} \mathrm{~T}\right)$ $\left(1 \mathrm{fT}=10^{-15} \mathrm{~T}\right)$, usually Brain electrical activities induce a few hundred fT. MEG imaging results can be superimposed on Magnetic Resonance Imaging(MRI) anatomical image of the subjects or patients, obtain neuromagnetic source imaging. MEG is currently approved for clinical use, mainly for epilepsy surgery, and preoperative brain imaging. In the United States, most MEG epilepsy centers uses it for preoperative imaging.

Beamformer is a spatial filter, the equivalent of a virtual sensor, the time course of brain can be derived from any source, commonly used to make MEG imaging. However, Beamformer usually imaging energy source positions, so the energy source is often weak 
cannot be identified, and sometimes missing source with important functions. Based on the results of the reconstruction beamformer, we propose a method of detecting weak source of functional connectivity (Functional Connectivity, FC) based. Through theoretical analysis and real data tests to demonstrate its effectiveness.

\section{Materials and Methods}

\section{Beamformer}

MEG can be modelled as

$$
\mathbf{X}(t)=\mathbf{A}(\theta) \mathbf{S}(t) .
$$

Among them, $\quad \mathbf{X}(t)$ indicates $\quad M$ (channels $) \times N($ sample points $)$ Matrix, $\mathbf{A}(\theta)$ indicates $M \times 2 p$ (grid points) The gain matrix $\mathbf{S}$, a $2 p \times N$ matrix, indicates the time course of the current dipole and $p$ the number of the assumed dipoles.

Beamformer estimated the time course of the location of the source $r$ by the following formula in $[2,3]$,

$$
\mathbf{s}(r, t)=\mathbf{W}^{T}(r) \mathbf{X}(t) .
$$

$\mathbf{W}^{T}(r)$ indicates the Spatial filter weights at the position r. Beamformer actually seek an optimized $\mathrm{W}$, so that it can pass the signal from $r$ while blocking signal from any other positions.. An implicit assumption is that the time course of different sources are not mutually completely correlated. Based on this assumption, we obtain the following solution[2, 3],

$$
\mathbf{W}(r)=\left[\mathbf{L}^{T}(r) \mathbf{R}^{-1} \mathbf{L}(r)\right]^{-1} \mathbf{L}^{T}(r) \mathbf{R}^{-1} .
$$

Wherein $\mathrm{R}$ is the correlation matrix of the data. In the position $\mathrm{r}$, the power of source can be expressed as,

$$
P(r)=\operatorname{tr}\left\{\left[\mathbf{L}^{T}(r) \mathbf{R}^{-1} \mathbf{L}(r)\right]^{-1}\right\} .
$$

$\operatorname{tr}\{\bullet\}$ indicates calculate the trace of "•". If we use $P(r)$ as an imaging index, you can get $3 \mathrm{D}$ source energy distribution, where the peaks usually was regarded as the sources.

\section{Weak Source Imaging Method based on FC}

By applying the equation (2) one can obtain each position waveform in source space. It seems like that a virtual electrode is inserted into each position within brain volume to pick up the waveforms. Energy distribution can identify active sources. However, in practical cases, some of the active source strength is relatively weak, this method does not distinguish the background activity. Assuming the source at location $\omega$ is identified. Correspondingly, its waveform will be $s(\omega)$. In general, waveforms of three directions of sources reconstructed by beamformer are completely coherent (perfectly correlated). Therefore, we only select the waveform in one direction for following analysis. In this study, the correlation coefficient between two sources is used to measure FC. Taking $\omega$ 
as the reference source location (seed point) and calculating the correlation.

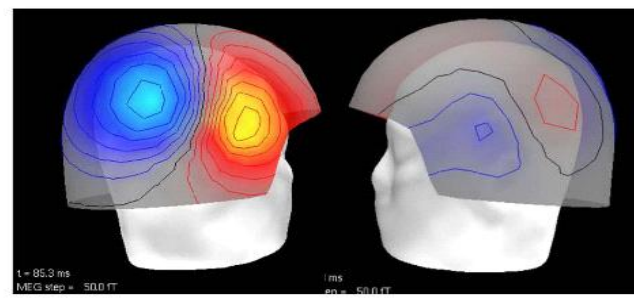

A

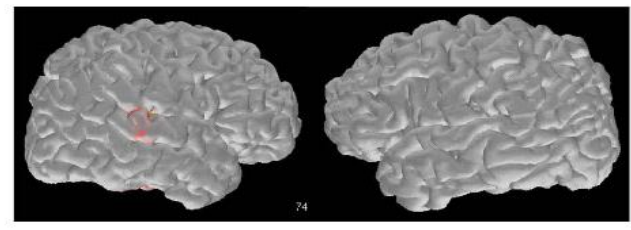

B

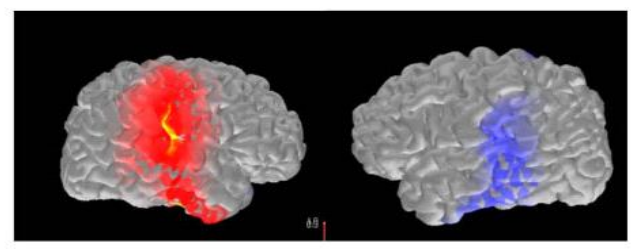

$\mathrm{C}$

Figure. 1. Cortical source localization and functional connectivity analysis of Auditory Evoked Fields

(AEF).

The stimulus is delivered to the left ear of the subject. Figure 1A; The magnetic field mapping on the scalp at latency $=83.5 \mathrm{~ms}$. Right temporal area shows strong field while left tiny. Figure 1B; Source activation on cortical surface. Right temporal cortex shows activation (noted that some activation are hidden in sulci) while left shows no activation. Figure 1C: Imaging results using in Eq. (5). Obviously, left temporal lobe has strong negative correlation with the seed (right temporal lobe).

Between $\mathbf{s}_{x}(\omega)$ and all the other points $\theta$ in the source space, we can obtain the following FC imaging index (FCI) at $\theta$,

$$
r_{(\omega, \theta)}=\frac{<\mathbf{s}_{x}(\omega), \mathbf{s}_{x}(\theta)>}{\left\|\mathbf{s}_{x}(\omega)\right\|\left\|\mathbf{s}_{x}(\theta)\right\|}
$$

Overlying $r_{(\omega, \theta)}$ on the anatomical images, one may get the weak source locations. With known locations, it is straightforward to obtain the waveforms by beamformer.

This method assumes that there are two sources $\omega$ and $\theta, \omega$ indicating strong source already identified and $\theta$ indicating weak source classical beamformer has difficulty in detecting. We assumes that two sources have highly correlated waveforms (such cases are common in primary brain cortices, for example, bilateral primary auditory cortices, bilateral primary visual cortices, bilateral motor and sensor cotices). It can be written as

$$
\left|r_{(\omega, \theta)}\right| \approx 1 \text {. }
$$

The power ratio of them can be expressed as 
$\frac{<\mathbf{s}(\omega)>}{n, \mathrm{n}-}>1$.

From equation (7), source at $\theta$ usually is neglected since it has very low power. Interestingly, the FCI of source $\omega$ and $\theta$ is $\left|r_{(\omega, \omega)}\right| \approx 1$ and $r_{(\omega, \theta)}=1$. Thus, the ratio of FCI between them will be

$$
\frac{r_{(\omega, \theta)}}{r_{(\omega, \omega)}} \approx 1 \text { or }-1
$$

For the locations other than $\omega$ or $\theta$, due to no correlation between test source and seed points, $\quad r_{(\omega, \theta)} \approx 0$. Thus, $\frac{r_{(\omega, \theta)}}{r_{(\omega, \omega)}} \approx 0$. It is straightforward that in the final source distribution image, there are two local peaks at locations $\omega$ and $\theta$, which can be readily recognized. However, classical beamformer only identifies one strong source and miss the weak one since it depends on source energy distribution. So, the proposed method can detect weak sources in the case where they have strong correlation with easily identified sources.

\section{Real Data Testing}

\section{Data Acquisition and Processing}

During the experiment, the pure tone stimuli $(1000 \mathrm{~Hz})$ was delivered to the left ear of the subjects (epilepsy patients). A total of 120 trials were collected. The experiment was approved by the Ethics Committee of Nanjing brain hospital, and the subjects signed an informed consent. Data were collected from the Elekta Neuromag 306-channel MEG Device. After data preprocessing and Artifact removal, a total average of 112 trials were used to get the final auditory evoked field data (Auditory Evoked Field, AEF). The MRI of the subjects were used to construct individual realistic MEG head model. Freesurfer was used to make segmentation and reconstruction of MRI data. The MRI were co-registered with MEG head using MNE-toolbox. The in-house code was used to conduct analysis.

\section{Results and Analysis}

Figure 1 shows the imaging result and FC analysis of AEF. Figure 1A Figure shows helmet mapping results of left ear auditory stimuli AEF. The latency is $83.5 \mathrm{~ms}$. Obviously, the right side of the head produced the strongest magnetic activity while on the left side only weak activity. FIG. 1B shows activation superimposed on the reconstructed cortical surface, which was obtained by beamformer. One can observe activated right temporal lobe (most hidden in the sulcus), while the left hardly has activation. The proposed method readily detected bilateral primary auditory cortices (Fig. 1C).

The results show that there is strong correlation between bilateral temporal lobes and they have negative correlation. That is, there is a 180 degree phase difference between 
them. Figure 2A shows on the right. Left panel of Figure 2 shows the superimposed waveforms of each sources, reconstructed by beamformer, within whole brain space. Figure $2 \mathrm{~B}$ shows at the peak time point the activations of right temporal lobe, which is overlaid on flatten cortical surface.

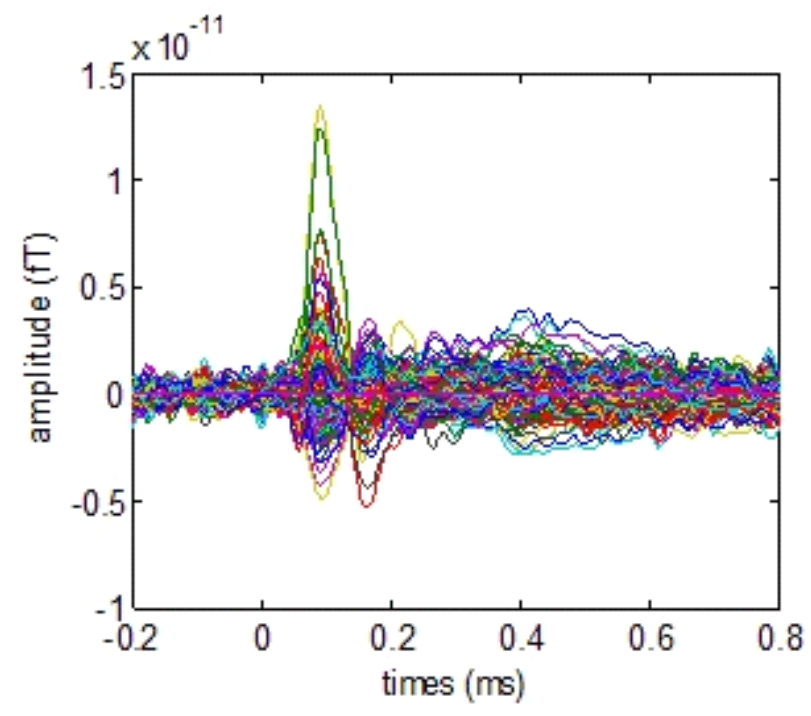

A
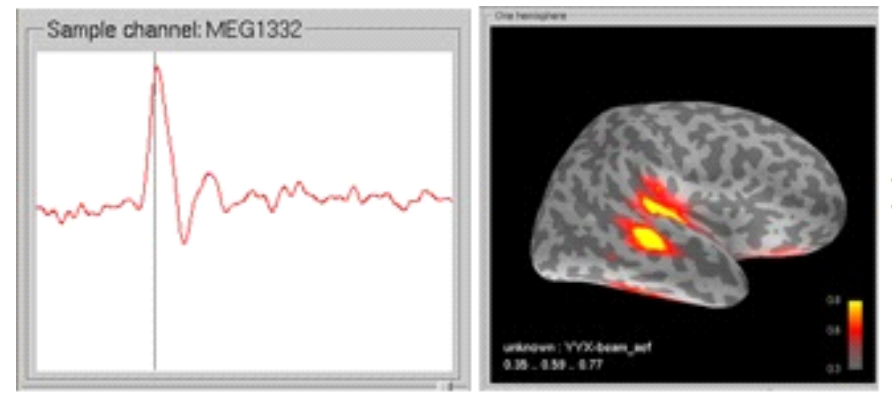

Figure 2. Cortical activations of AEF overlaid on flatten cortical surface.

Left panel shows the AEF waveform with all the channels superimposed. The vertical line indicates the peak time point with a latency of $85.3 \mathrm{~ms}$. Right panel shows the activations on flatten cortical surface.

\section{Discussion and Conclusion}

Aimed at the problem where classical beamformer has difficulty in identifying weak sources, we proposed a method to detect such sources based on intersource functional connectivity. This approach takes good use of the fact that, although the weak sources have small power, they have strong correlation with easily identified sources. For a system, there have not to have strong correlation between sources. However, for brain, correlation between brain regions widely exists since brain conducts various tasks by interaction and coupling between many functional regions. In this study we proposed a new method to detect weak sources. AEF was used to prove its correctness. We also did some theoretical analysis on the principle of the proposed method. Further development 
and extension of this method is our next task.

\section{References}

[1] Hari, R. and R. Salmelin, Magnetoencephalography: From SQUIDs to neuroscience. Neuroimage 20th anniversary special edition. Neuroimage, 2012. 61(2): p. 386-96.

[2] Van Veen, B.D., et al., Localization of brain electrical activity via linearly constrained minimum variance spatial filtering. IEEE Trans Biomed Eng, 1997. 44(9): p. 867-80.

[3] Sekihara, K., et al., Reconstructing spatio-temporal activities of neural sources using an MEG vector beamformer technique. IEEE Trans Biomed Eng, 2001. 48(7): p. 760-71. 